\title{
A new beginning for Acta Mechanica Sinica
}

\author{
Xiaojing Zheng ${ }^{1}$
}

Published online: 10 March 2021

(C) The Chinese Society of Theoretical and Applied Mechanics and Springer-Verlag GmbH Germany, part of Springer Nature 2021

Welcome to the first issue of Acta Mechanica Sinica (AMS) of 2021.

From this issue, AMS starts to publish monthly and enriches its columns with Rapid Communication, Perspective, News and Views, Extreme Mechanics, X-Mechanics, and Distinguished Young Scholars, in addition to previous Fluid Mechanics, Solid Mechanics, Dynamics, Vibration and Control, and Biomechanics.

As the newly appointed Editor-in-Chief of AMS, together with Xuesong Wu from Imperial College London, UK and Zhigang Suo from Harvard University, USA, we are joined by an extraordinary team of Associate Editors-in-Chiefs and Editors. The new Editorial Board strives for fast publication for high impact works and hence comes up with the newly "Aims and Scope" of AMS.

As the flagship journal of the Chinese Society of Theoretical and Applied Mechanics (CSTAM) with over 36,000 society members, AMS publishes top research work in the global mechanics community. The Journal focuses on mechanics and related issues when solving key engineering and scientific problems, especially problems concerned with energy security, human health, and other common human challenges. It reports the latest developments in mechanics including solid mechanics, fluid mechanics, dynamics and control, biomechanics, etc. It explores analytical, computational and experimental progresses in all areas of mechanics. The Journal also encourages research in interdisciplinary subjects, and serves as a bridge between mechanics and other branches of engineering and sciences.

The work published in AMS must extend the current knowledge in mechanics through new discoveries and novel results in mechanics. Such as new theories or great extension of existing ones, new algorithms in computation, and novel experimental techniques.

Facing these new changes, we are confident that AMS will serve the mechanics community better by working on ways to publish papers in high quality.

Xiaojing Zheng

Lanzhou University

China

Xiaojing Zheng

xjzheng@1zu.edu.cn

1 Key Laboratory of Mechanics on Western Disaster and Environment, Lanzhou University, Lanzhou 730000,

China 